\title{
A Computational Dynamic Trust Model for User Authorization
}

\author{
Chang Young Jung1), V. Bhava Keerthana2)
}

\begin{abstract}
Development of authorization mechanisms for secure data access by an outsized community of users in Associate in Nursing open atmosphere is a crucial downside within the ever-growing web world. During this paper we have a tendency to propose a procedure dynamic trust model for user authorization, nonmoving in findings from scientific discipline. In contrast to most existing procedure trust models, this model distinguishes trusting belief in integrity from that in ability totally in numerous contexts and accounts for sound judgment within the analysis of a selected trustee by different trustors. Simulation studies were conducted to match the performance of the projected integrity belief model with alternative trust models from the literature for various user behavior patterns. Experiments show that the projected model achieves higher performance than alternative models particularly in predicting the behavior of unstable users.
\end{abstract}

Keywords: trust model, authorization, crucial, scientific, ability, integrity, literature.

\section{Introduction}

The everyday increasing wealth of knowledge out there on-line has created secure info access mechanisms an essential a part of information systems these days[1-3]. The thought analysis efforts for user authorization mechanisms in environments wherever a possible user's permission set isn't predefined, principally concentrate on role-based access management (RBAC), that divides the authorization method into the role-permission and user-role assignment[4-10]. RBAC in fashionable systems uses digital identity as proof a couple of user to grant access to resources the user is entitled to.

Received(July 7, 2015), Review Result(1st: July 24, 2015, 2nd: August 21, 2015), Accepted(December 10, 2015)

${ }^{1}$ Graduate School of Advanced Imaging \& Multimedia Chung-Ang University 84, Heukseok-ro, Dongjak-gu, Seoul, Korea email: mywood@naver.com

${ }^{2}$ (Corresponding Author) Department of Computer Science and Engineering, KL University email: keerthanakeerthi@gmail.com 


\section{Trust Model}

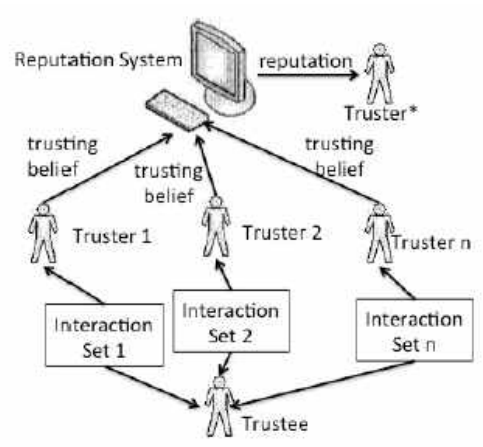

[Fig. 1] Architecture Diagram

Holding proof doesn't essentially certify a user's smart behavior. So we propose a procedure dynamic trust model for user authorization. Mechanisms for building trusting belief mistreatment the first-hand (direct experience) also as second-hand info (recommendation and reputation) area unit integrated into the model. The contributions of the model to procedure trust literature are:

- The model is frozen in findings from science, i.e. it provides machine-controlled trust management that mimics trusting behaviors within the society, transferral trust computation for the digital world nearer to the analysis of trust within the planet.

- not like different trust models within the literature, the projected model accounts for various sorts of trust. Specifically, it distinguishes trusting belief in integrity from that in ability.

- The model takes under consideration the judgement of trust ratings by completely different entities, and introduces a mechanism to eliminate the impact of judgement in name aggregation.

We implemented this model with

1. Mcknight's Trust Model

2. Procedure Trust Models

3. Context and Trusting Belief

4. Belief info and reputation Aggregation ways 


\subsection{Mcknight's Trust Model}

The social trust model, that guides the look of the procedure model during this paper, was projected by McKnight et al. when measurement over sixty papers across a good vary of disciplines. it's been valid via empirical study. This model defines 5 abstract trust types: trusting behavior, trusting intention, trusting belief, institution-based trust, and disposition to trust. Trusting behavior is associate action that will increase a protagonist's risk or makes the truster susceptible to the trustee. Trusting intention indicates that a protagonist is willing to have interaction in trusting behaviors with the trustee. A trusting intention implies a trust call and ends up in a trusting behavior. Two subtypes of trusting intention are:

1. disposition to depend: the willing state to create oneself susceptible to the trustee.

2. Subjective likelihood of relying.

\subsection{Computational Trust Models}

The problem of creating and maintaining dynamic trust has attracted several analysis efforts. one in every of the primary makes an attempt making an attempt to formalize trust in applied science was created by Marsh. The model introduced the ideas wide utilized by different researchers like context and situational trust. several existing name models and security mechanisms believe a social network structure. Propose associate approach to extract name from the social topology that encodes name info. Walter et al. proposed a dynamic trust model for social networks, supported the construct of feedback spatial relation. The model, that allows computing trust between 2 disconnected nodes within the network through their neighbor nodes, is appropriate for application to recommender systems. Lang proposes a trust model for access management in P2P networks, supported the belief of transitivity of trust in social networks, wherever a straightforward mathematical model supported fuzzy set membership is employed to calculate the trait of every node in an exceedingly trust graph figuration interactions between network nodes.

\subsection{Context and Trusting Belief}

Trust is environment-specific . Each trusters concern and trustees' behavior vary from one state of affairs to a different. These things area unit known as contexts. A protagonist will 
specify the minimum trusting belief required for a particular context. Direct expertise info is maintained for every individual context to hasten belief change. During this model, a protagonist has one integrity trust per trustee altogether contexts. If a trustee disappoints a protagonist, the wrongful conduct lowers the truster's integrity belief in him. For integrity trust, contexts don't have to be compelled to be distinguished. Competence trust is context-dependent. the actual fact that Bob is a wonderful academician doesn't support to trust him as a chief. A illustration is devised to spot the ability sort and level required in an exceedingly context.

\subsection{Belief info and name Aggregation methods}

Belief a couple of trustee's ability is context specific. A trustee's ability changes comparatively slowly with time. Therefore, ability ratings allotted to her area unit viewed as samples drawn from a distribution with a gradual mean and variance. ability belief formation is developed as a parameter estimation drawback. data point ways area unit applied on the rating sequence to estimate the steady mean and variance, that area unit used because the belief worth concerning the trustee's ability and therefore the associated sure thing.

\section{System Configuration: HARDWARE REQUIREMENTS:}

Hardware: Pentium

Speed: $1.1 \mathrm{GHz}$

RAM: $1 \mathrm{~GB}$

Hard Disk: 20 GB

Floppy Drive: $1.44 \mathrm{MB}$

Key Board: Standard Windows Keyboard

Mouse: Two or Three Button Mouse

Monitor: SVGA

\section{SOFTWARE REQUIREMENTS:}

Operating System: Windows

Technology: Java and J2EE

Web Technologies: Html, Java Script, CSS

IDE : My Eclipse

Web Server: Tomcat 
Tool kit: Android Phone

Database: My SQL

Java Version: J2SDK1.5

\section{Conclusion}

This paper we've got an inclination to propose a procedure dynamic trust model for user authorization, static in findings from discipline. In distinction to most existing procedure trust models, this model distinguishes trusting belief in integrity from that in ability altogether in varied contexts and accounts for sound judgement at intervals the analysis of a particular trustee by completely different trusters. Simulation studies were conducted to match the performance of the projected integrity belief model with different trust models from the literature for varied user behavior patterns. Experiments show that the projected model achieves higher performance than different models notably in predicting the behavior of unstable users.

\section{References}

[1] Y. Zhong, B. Bhargava, Y. Lu, and P. Angin, A Computational Dynamic Trust Model for User Authorization, IEEE TRANSACTIONS ON DEPENDABLE AND SECURE COMPUTING, (2015), Vol.12, No.1, pp.1-15.

[2] A. Das and M. M. Islam, Secured Trust: A Dynamic Trust Computation Model for Secured Communication in Multiagent Systems, IEEE Trans. Dependable and Secure Computing, (2012), Vol.9, No.2, pp.261-274.

[3] D. McKnight, V. Choudhury, and C. Kacmar, Developing and Validating Trust Measures for E-Commerce: An Integrative Topology,” Information Systems Research, (2002), Vol.13, No.3, pp.334-359.

[4] A. Nagarajan and V. Varadharajan, Dynamic Trust Enhanced Security Model for Trusted Platform Based Services, Future Generation Computer Systems, (2011), Vol.27, pp.564-573.

[5] J. Sabater and C. Sierra, Social ReGreT, a Reputation Model Based on Social Relations, ACM SIGecom Exchanges, (2002), Vol.3, No.1, pp.44-56.

[6] O. A. Wahab, J. Bentahar, H. Otrok, and A. Mourad, A survey on trust and reputation models for Web services: Single, composite, and communities, Decision Support Systems, (2015), Vol.74, pp.121-134.

[7] Z. Maamar, S. Subramanian, P. Thiran, D. Benslimane, and J. Bentahar, An approach to engineer communities of web services: concepts, architecture, operation, and deployment, International Journal of E-Business Research, (2009), Vol.5, No.4, pp.1-21.

[8] K. Voevodski, M. F. Balcan, H. Rglin, S. H. Teng, and Y. Xia, Active clustering of biological sequences, Journal of Machine Learning Research, (2012), Vol.13, No.1, pp.203-225. 
[9] A. A. Selcuk, E. Uzun, and M. R. Pariente, A review on computational trust models for multi-agent systems, International Journal of Network Security, (2004), Vol.6, No.3, pp.235-245.

[10] B. Khosravifar, J. Bentahar, A. Moazin, P. Thiran, Analyzing communities of web services using incentives, International Journal of Web Services Research, (2010), Vol.7, No.3, pp.30-51. 